\title{
SLIDING-VIBRATING RESPONSES OF ELASTIC STRUCTURES
}

\author{
by Fu Yu-An*
}

\begin{abstract}
SUMMARY
By using simulated friction forces, analytical expressions were derived from the sliding-vibrating response of a single degree of freedom system under harmonic excitation or the "disadvantageous period reciprocating motion", taking the mass of the sliding base into consideration. Some of the general laws were studied and some new characteristics determined which had previously been ignored by assuming rigid body motion. The analysis methods adopted in this paper have been confirmed in comparison with the results of model tests on a shake table.
\end{abstract}

\section{INTRODUCTION}

Many possible ways of safeguarding buildings and construction from earthquake disasters have been studied at different times. Recently, base-sliding isolation (BSI) measures have come to be used around the world even though more research is still needed to apply the technique in practice. Most of the research to date has has been based on an assumption of rigid body behaviour which has resticted our understanding of a structures slidingvibrating response (SSVR).

During the 7th WCEE in 1980, Professor A.S. Arya of Roorkee University in India was the first to publish results of model tests on BSI based on an elastic body assumption. At the 8th WCEE in 1984, he advanced a numerical method for determining the sliding-vibrating response for a single degree of freedom system under earthquake excitation, taking the mass of the sliding base into consideration. In china, a similar numerical method was also' developed in 1980.

In general, numerical methods are expensive, take considerable computer time and make it difficult to determine general laws. In order to completely determine the mechanism of BSI and its significant parameters, it was necessary to develop suitable analytical methods to calculate the the sliding-vibrating response of elastic structures.

\footnotetext{
* Research Engineer, Central Institute of Building \& Construction, Ministry of
} Metallurgical Industry, Beijing, China.
In this paper, simulated friction forces are used to derive the analytical expressions for the sliding-vibrating response of a single degree of freedom system under harmonic excitation or the "disadvantageous period reciprocating motion", taking the mass of the sliding base into consideration. The general laws were studied and some new characteristics determined. The analytical methods presented in this paper have confirmed by comparison with the results of model tests carried out on a shake table.

\section{EQUATION OF MOTION AND ITS PRIMARY ANALYSIS}

In general, in calculating the slidingvibrating response of a single degree of freedom system, it is possible to neglect the mass of sliding base. However, for the analyses to be described herein, the structure was idealised as a special two degree of freedom discrete mass model as shown in Fig. 1.

The differential equations of motion for this structure are as follows:

$$
\begin{gathered}
\mathrm{m}_{1} \ddot{\mathrm{u}}_{1}+\mathrm{c}\left(\dot{\mathrm{u}}_{1}-\dot{\mathrm{u}}_{2}\right)+\mathrm{k}\left(\mathrm{u}_{1}-\mathrm{u}_{2}\right)=0 \\
\mathrm{~m}_{2} \ddot{\mathrm{u}}_{2}+\mathrm{c}\left(\dot{\mathrm{u}}_{2}-\dot{\mathrm{u}}_{1}\right)+\mathrm{k}\left(\mathrm{u}_{2}-\mathrm{u}_{1}\right)=\mathrm{F}(\mathrm{t}) \\
\text { where } \mathrm{u}_{1}, \dot{\mathrm{u}}_{1}, \dot{\mathrm{u}}_{1}: \begin{array}{l}
\text { the absolute displacement, } \\
\text { velocity and acceleration } \\
\text { of top mass } \mathrm{m}_{1} \text { respect- } \\
\text { ively; }
\end{array}
\end{gathered}
$$




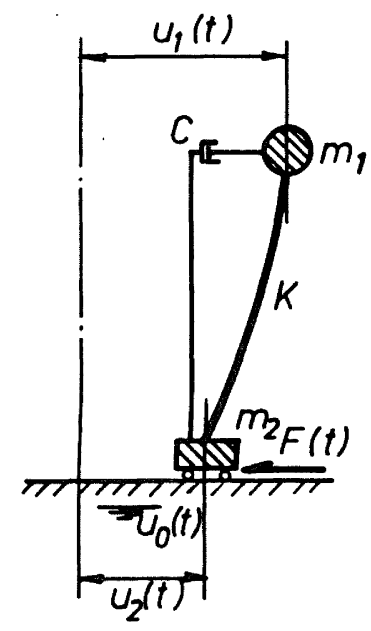

FIG. 1 CALCULATING SCHEME

$$
\begin{aligned}
\mathrm{u}_{2}, \dot{\mathrm{u}}_{2}, \ddot{\mathrm{u}}_{2}: \begin{array}{l}
\text { : } \\
\text { the absolute displacement, } \\
\text { velocity and acceleration } \\
\text { of top mass } \mathrm{m}_{2} \text { respect- } \\
\text { ively; }
\end{array} \\
\mathrm{F}(\mathrm{t}) \quad \begin{array}{l}
\text { : the shear force at the } \\
\text { bottom of sliding base }
\end{array}
\end{aligned}
$$

The shear force at the bottom of structure is just the friction force acting on the sliding face through which various vibration inputs control over SSVR. According to Coulomb's friction law, the friction force for dry friction follows the relation:

$$
|F(t)| \leq\left(m_{1}+m_{2}\right) g \mu
$$

where $g$ : gravitational acceleration;

$\mu:$ coefficient of friction,

Adding equation (1) to (2) we obtain:

$m_{1} \ddot{u}_{1}+m_{2} \ddot{u}_{2}=F(t)$

Then $\left|m_{1} \ddot{u}_{1}+m_{2} \ddot{u}_{2}\right| \leq\left(m_{1}+m_{2}\right) g \mu$

This shows that the algebraic sum of all quantities of inertia acting on the structure is always less than or equal to the maximum friction force at the bottom of the base of the sliding-vibrating structure.

If the structure acts as a rigid body, then $\ddot{\mathrm{u}}_{1}=\ddot{\mathrm{u}}_{2}=\ddot{\mathrm{u}}$, and by substituting into equation (5), we have:

$$
|u(t)| \leq g \mu
$$

This conclusion is borne out by the results of tests on rigid body sliding-vibrating responses.

If the structure acts as an elastic body, taking no account of the mass of the sliding base, i.e. $\mathrm{m}_{2}=0$, then from equation (5) we have:

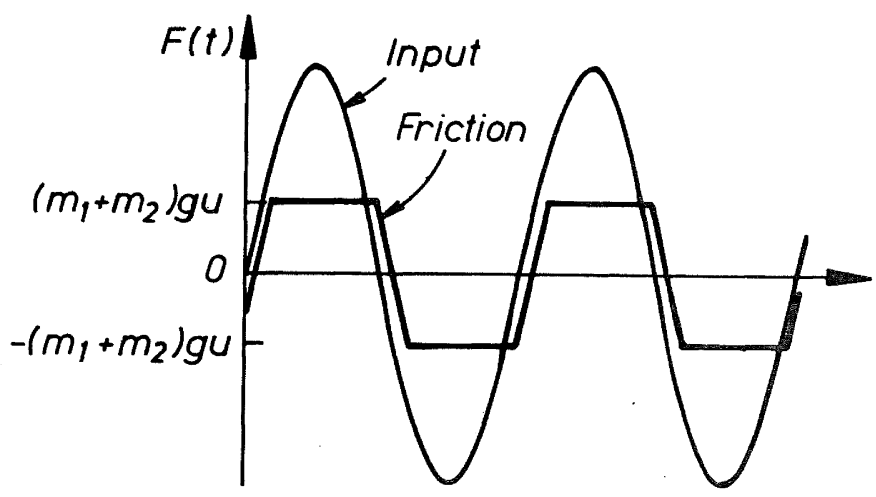

FIG. 2 FRICTION CURVE UNDER HARMONIC INPUT

$$
\left|\ddot{u}_{1}(t)\right| \leq g \mu
$$

Therefore, neglecting the effect of the mass of the sliding base upon the structure, the sliding-vibrating response of the single degree of freedom elastic system is the same as if it were a rigid body.

obviously, the rigid body is a special case of an elastic body. The structure without base mass is assumed only for its theoretical significance, which does not exist in practice. In order to analyse the SSVR exactly, it is necessary to take the mass of the sliding base into consideration as follows.

\section{CALCULATION OF SSVR UNDER HARMONIC \\ EXCITATION}

Under the action of a harmonic wave, the friction time-history of SSVR in stable state is similar to a harmonic wave curve with the peaks cut off as shown in Fig. 2. To simplify the analysis, a trapezoid square wave was used to simulate this kind of friction time-history. Therefore the friction force curve under a harmonic excitation with a period, T, can be expressed by the wave shown in Fig. 3 and its Fourier series can be indicated in the form:

$F(t)=\frac{4 g \mu}{\alpha \pi}\left(m_{1}+m_{2}\right) \sum \frac{\operatorname{SIN}(n \alpha) \cdot \operatorname{SIN} \frac{2 n \pi t}{T}}{n}$

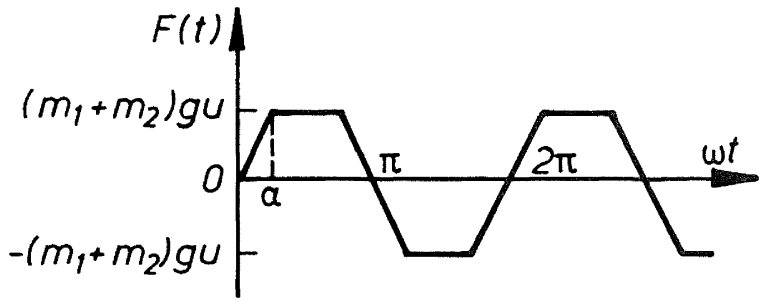

FIG. 3 SIMULATION OF FRICTION CURVE 
Substituting equation (8) into (2) and introducing the differential operator $D$ (where $D^{n}=a^{n} / a t^{n}$ ), after the operation and simplification of equation (1) and (2) we have:

$$
\begin{gathered}
{\left[m_{1} m_{2} D^{4}+C\left(m_{1}+m_{2}\right) D^{3}+K\left(m_{1}+m_{2}\right) D^{2}\right] u_{1}} \\
=(C D+K) \frac{4 g \mu}{\alpha \pi}\left(m_{1}+m_{2}\right)\left[\frac{\operatorname{SIN}(n \alpha) \operatorname{sIN} \frac{2 n \pi t}{T}}{n^{2}}\right. \\
n=1,3,5 \ldots .
\end{gathered}
$$

Let $\mathrm{D}^{2} \mathrm{u}_{1}$, the absolute acceleration $\ddot{u}_{1}$ of top mass $m_{1}$, be a new variable unit and denote it by $Y$, then equation (9) can be rewritten as follows:

$$
\begin{aligned}
& Y+\frac{m_{1}+m_{2}}{m_{2}} \frac{C}{m_{1}} \dot{Y}+\frac{m_{1}+m_{2}}{m_{2}} \frac{K}{m_{1}} Y \\
& =\frac{8 g \mu}{\alpha T}\left(\frac{m_{1}+m_{2}}{m_{2}}\right) \frac{c}{m_{1}} \sum \frac{\operatorname{SIN}(n \alpha) \operatorname{SIN} \frac{2 n \pi t}{T}}{n} \\
& n=1,3,5 \ldots \\
& +\frac{4 g \mu}{\alpha \pi}\left(\frac{m_{1}+m_{2}}{m_{2}}\right) \frac{K}{m_{1}} \sum \frac{\operatorname{SIN}(n \alpha) \operatorname{SIN} \frac{2 n \pi t}{T}}{n^{2}} \\
& n=1,3,5 \ldots
\end{aligned}
$$

The special solution of equation (10) is just the sliding-vibrating absolute acceleration response of the top mass in the stable state.

Introduce

$$
\begin{aligned}
& \frac{\mathrm{c}}{\mathrm{m}_{1}}=2 \mathrm{p} \xi \\
& \frac{\mathrm{k}}{\mathrm{m}_{1}}=\mathrm{p}^{2} \\
& \frac{\mathrm{m}_{1}+\mathrm{m}_{2}}{\mathrm{~m}_{2}}=\mathrm{M}
\end{aligned}
$$

where $p$ : the inherent circular frequency;

$\xi$ : the damping ratio of structure where base is fixed without sliding.

Then the special solution of equation (10) can be easily obtained:

$$
\begin{gathered}
\mathrm{Y}=\ddot{\mathrm{u}}_{1}=\sum\left(\mathrm{A} \cdot \cos \frac{2 \mathrm{n} \pi t}{\mathrm{~T}}+\mathrm{B} \cdot \operatorname{SIN} \frac{2 \mathrm{n} \pi t}{\mathrm{~T}}\right) \\
\mathrm{n}=1,3,5 \ldots .
\end{gathered}
$$

where

$$
A=\frac{-64 g \mu \pi^{2} M T \xi p n \cdot \operatorname{SIN}(n \alpha)}{\alpha\left(16 M^{2} \pi^{3} T^{2} \xi^{2} p^{2} n^{2}+\left(M T^{2} p^{2}-4 \pi^{2} n^{2}\right)^{2}\right)}
$$

$$
\begin{aligned}
& B= \\
& \frac{4 g \mu T^{2} \operatorname{SIN}(n \alpha)\left(16 M^{2} \pi^{2} \xi^{2} p^{2} n^{2}+M p^{2}\left(M T^{2} p^{2}-4 \pi^{2} n^{2}\right)\right)}{\alpha \pi n^{2}\left(16 M^{2} \pi^{2} T^{2} \xi^{2} p^{2} n^{2}+\left(M T^{2} p^{2}-4 \pi^{2} n^{2}\right)^{2}\right)}
\end{aligned}
$$

This is the analytical expression for SSVR in Fourier series form. $\alpha$ is a fundamental parameter whereby the shape of trapezoid square wave of friction can be obtained; it is related to the moment of sliding and can be found by the following equation:

$$
\alpha=\arcsin \left(\frac{g \mu}{B A_{O}}\right)
$$

where $A_{0}$ : input peak value of harmonic wave;

B : the dynamic amplification factor of structure with fixed base.

$$
=\frac{1}{\sqrt{\left(1-\frac{\omega^{2}}{p^{2}}\right)^{2}+\left(2 \xi \frac{\omega}{p}\right)^{2}}}
$$
where $\omega$ : the circular frequency of input
vibration.

Equations (14) to (18) can be used to calculate the SSVR in a stable state under various harmonic excitations. Of course if the input is obtained without sliding, i.e. $\ddot{u}_{I}(t)_{\max }<g \mu$, we need not calculate the SSVR. In this case, the structure acts as if it had a fixed base and its vibration response can be calculated by the theory of dynamics of structures.

\section{CALCULATION OF SSVR UNDER DISADVANTAGEOUS \\ PERIOD RECIPROCATING EXCITATION}

The so-called "disadvantageous period reciprocating input" is referred to as input where the period approaches that of the structure (resoriance frequency) or whose peak value is correspondingly high. If this input is just a harmonic wave, we can, of course, solve it by the previous formulas. Since the input isn't always harmonic, it is necessary to develop a more general method to calculate the SSVR.

Under the "disadvantageous period reciprocating motion", the base of structure appears to be in continuous reciprocation. In this case, the friction time-history can be simulated by a rectangular square wave (Fig. 4) and its Fourier series form can be expressed as follows:

$$
F(t)=\frac{4 g \mu}{\pi}\left(m_{1}+m_{2}\right) \sum \frac{\sin \frac{2 n \pi t}{T}}{n}
$$

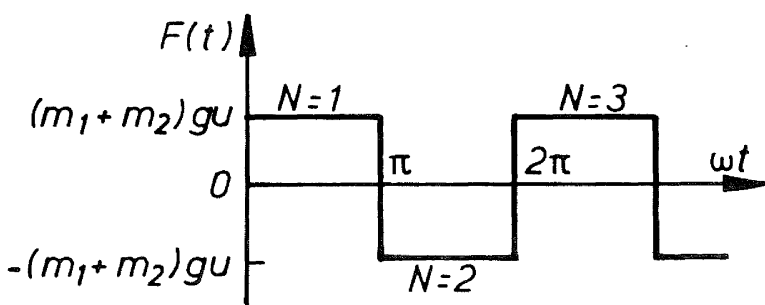

FIG. 4 SIMULATION OF FRICTION CURVE 
Substituting equation (19) into (2), after operation as above mentioned, the formula which denotes the analytical result of the SSVR can be derived:

$\ddot{u}_{1}=\sum\left(A \cdot \cos \frac{2 n \pi t}{T}+B \cdot \sin \frac{2 n \pi t}{T}\right)$

$$
\mathrm{n}=1,3,5 \ldots
$$

where

$A=\frac{-64 g \mu \pi^{2} N T \xi p n^{2}}{16 \pi^{2} M^{2} T^{2} \xi^{2} p^{2} n^{2}+\left(M T^{2} p^{2}-4 \pi^{2} n^{2}\right)^{2}}$

$B=\frac{4 g \mu T^{2}\left(16 M^{2} \pi^{2} \xi^{2} p^{2} n^{2}+M p^{2}\left(M p^{2} T^{2}-4 \pi^{2} n^{2}\right)\right)}{\pi n\left(16 \pi^{2} M^{2} T^{2} \xi^{2} p^{2} n^{2}+\left(M T^{2} p^{2}-4 \pi^{2} n^{2}\right)^{2}\right)}$

Formula (20) can be also derived by taking the limit formula (14) as $\alpha$ approaches zero. Obviously, a trapezoid square wave for the friction force curve will certainly approximate a rectangular square wave as a approaches zero. Therefore it is expected that the limiting result of formula (14) should be equal to formula (20).

Similarly, the analytical expression for SSVR under the disadvantageous period reciprocation input will be derived by Duhamel integration instead of the Fourier series method in order to develop the general laws of SSVR. Here equations (1) and (2) can be rewritten as follows:

$$
\begin{aligned}
& m_{1} \ddot{\delta}_{1}(t)+C \dot{\delta}(t)+K \delta(t)=0 \\
& m_{2} \ddot{\delta}_{2}(t)-c \dot{\delta}(t)-K \delta(t)=F(t)
\end{aligned}
$$

From equations (23) and (24) we have:

$\ddot{\delta}(t)+\frac{m_{1}+m_{2}}{m_{2}} \frac{c}{m_{1}} \dot{\delta}(t)+\frac{m_{1}+m_{2}}{m_{2}} \cdot \frac{k}{m_{1}} \delta(t)=\frac{-F(t)}{m_{2}}$

where $\delta(t), \dot{\delta}(t), \ddot{\delta}(t)$ are the relative displacement, velocity and acceleration respectively between top and base.

$$
\text { Let } \quad \begin{aligned}
\frac{\mathrm{c}}{\mathrm{m}_{1}} & =2 \rho \xi \\
\frac{\mathrm{K}}{\mathrm{m}_{1}} & =\mathrm{p}^{2}
\end{aligned}
$$

the solution of equation (25) can be given in a form of Duhamel integration.

$$
\begin{gathered}
\delta(t)=\frac{1}{\sqrt{1-\frac{m_{1}+m_{2}}{m_{2}} \xi^{2}} \cdot \sqrt{\frac{m_{1}+m_{2}}{m_{2}} p}} . \\
\int^{t} \frac{F(t)}{m_{2}} \cdot e^{-\frac{m_{1}+m_{2}}{M_{2}} p \xi(t-\tau)} \cdot \operatorname{sIN} \sqrt{1-\frac{m_{1}+m_{2}}{m_{2}}} \xi^{2} . \\
. \sqrt{\left.\frac{m_{1}+m_{2}}{m_{2}} p(t-\tau)\right) d \tau}
\end{gathered}
$$

The rectangular square wave curve can also be rewritten:

$$
\begin{aligned}
& F(t)=(-1)^{N-1}\left(m_{1}+m_{2}\right) g \mu \\
& \text { for }\left(\frac{N-1}{2} T \leq t<\frac{N T}{2}\right)
\end{aligned}
$$

Set

$$
\begin{aligned}
& \mathrm{n}=\sqrt{1-\frac{\mathrm{m}_{1}+\mathrm{m}_{2}}{m_{2}}} \xi^{2} \cdot \sqrt{\frac{m_{1}+m_{2}}{m_{2}}} \mathrm{p} \\
& \mathrm{a}=\frac{\mathrm{m}_{1}+\mathrm{m}_{2}}{\mathrm{~m}_{2}} \mathrm{p} \xi
\end{aligned}
$$

Substituting equations (29) to (31) into (28), after operations of integration and sorting and merging, the sliding-vibrating relative displacement response of the top mass can be derived as:

$$
\begin{gathered}
\delta(t)=-\frac{g \mu}{p^{2}}\left[1-e^{-a t} \cdot \frac{1}{\cos \varepsilon} \cos (n t-\varepsilon)\right] \\
\text { for }\left(0 \leq t<\frac{T}{2}\right)
\end{gathered}
$$

and

$$
\begin{aligned}
& \delta(t)=-\frac{g \mu}{p^{2}}\left\{(-1)^{N-1}-\frac{e^{-a t}}{\cos \varepsilon} \cos (n t-\varepsilon)\right. \\
& +2 \sum_{i=1}^{N-1}(-1)^{i-1} \cdot e^{-a\left(t-\frac{i T}{2}\right)} \\
& \text { - } \left.\frac{1}{\cos \varepsilon} \cos \left[n\left(t-\frac{i T}{2}\right)-\varepsilon\right]\right\} \\
& \text { for }\left(\frac{N-1}{2} T \leq t<\frac{N T}{2}, N>I\right)
\end{aligned}
$$

where

$$
\begin{aligned}
& \cos \varepsilon=\sqrt{1-\frac{m_{1}+m_{2}}{m_{2}}} 2 \\
& \sin \varepsilon=\sqrt{\frac{m_{1}+m_{2}}{m_{2}}}
\end{aligned}
$$

The general solution for the absolute acceleration response of the top mass can be finally derived as:

$$
\begin{aligned}
\ddot{u}_{1}(t) & =g \mu\left\{(-1)^{N-1}+\frac{e^{-a t}}{\cos \varepsilon} \cos \left(n t-\varepsilon-\varepsilon^{\prime}\right)\right. \\
- & 2 \sum_{i=1}^{N-1}(-1)^{i-1} \cdot \frac{e^{-a\left(t-\frac{i T}{2}\right)}}{\cos \varepsilon} \\
& \left.\left.\cdot \cos \left[n\left(t-\frac{i T}{2}\right)-\varepsilon-\varepsilon^{\prime}\right)\right]\right\}
\end{aligned}
$$

where

$$
\begin{aligned}
& \cos \varepsilon^{\prime}=\frac{2 a \xi}{p}-1 \\
& \sin \varepsilon^{\prime}=\frac{2 n \xi}{p}
\end{aligned}
$$

Formula (36) is the analytical expression for SSVR from the Duhamel integration. Its value equals to that given by formula (20) using Fourier series. 


\section{VERIFICATION THROUGH TESTS}

In this paper, when calculating SSVR, a friction simulation was adopted as input stead of the excitation wave itself; this made it possible to calculate SSVR by an analytical method. How about this treatment? Is it reliable? It must be verified through tests. Model tests of SSVR were carried out on a shake table which was an electromagnetic type with $300 \mathrm{~kg}$ thrust. The specimen was made of four pieces of small angle iron, a steel top block and a sliding base of graphite plates. The. scheme of the test is shown in Fig. 5 and the parameters of the specimen are listed in Table 1. TABLE 1

\begin{tabular}{|ccccc|}
\hline$m_{1}(\mathrm{~kg})$ & $m_{2}(\mathrm{~kg})$ & $p(\mathrm{~Hz})$ & $\xi$ & $\mu$ \\
\hline 29.5 & 1.75 & $2 \mathrm{x}_{2}{ }^{7}$ & 0.05 & 0.20 \\
\hline
\end{tabular}

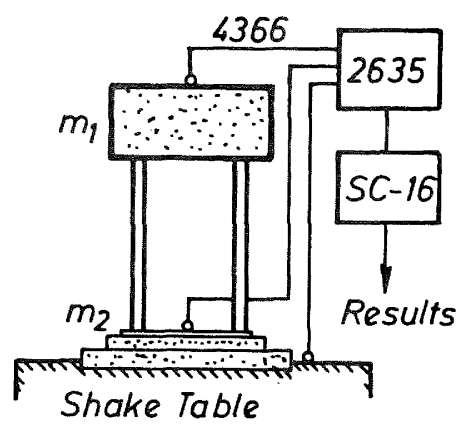

FIG. 5 SCHEME OF TEST

During the tests, the shaking used was of a steady-state sinusoidal type with a peak value of $200 \mathrm{gal}$ and a period of 0.143 sec which was equal to the natural period of the specimen. In this case, a continual reciprocating sliding took place at the base. The acceleration time-history measured at the top mass of the specimen is shown in Fig. $6 \mathrm{a}$.

The analytical result of the SSVR for this specimen can be calculated in forms of either harmonic wave input or disadvantageous period reciprocating input. Programming formula (14) for the trapezoid Fourier method (TFM), formula (20) for the rectangular Fourier method (RFM) and formula (36) for the rectangular Duhamel method (RDM) into the computer programmes, and substituting the parameters of the specimen into these programmes, the analytical curves of Fig. $6(b-d)$ were obtained.

In a Masters degree thesis entitled "Analysis on Sliding-Friction Isolation of Structures", 8 sets of model shake table

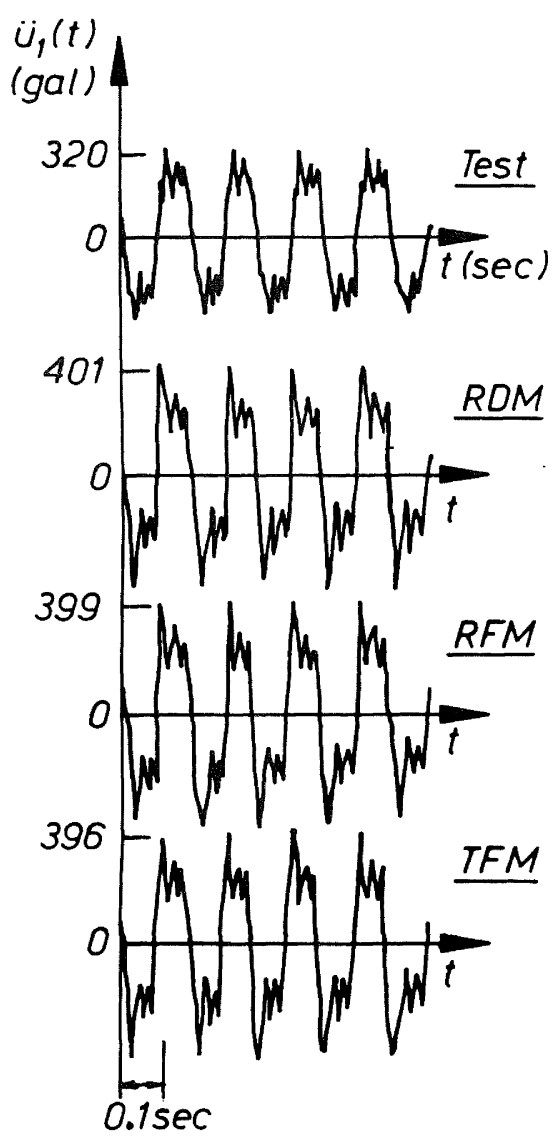

FIG. 6 COMPARISON OF SSVR (1) input: $A=200 \quad \mathrm{~T}=0.143$

test results on SSVR under the harmonic shaking inputs were given; also given were the analytical results of these tests by the numerical method. For these tests, the parameters of each specimen were substituted into the analysis methods and the calculated results and acceleration time histories are compared in Table 2 and Figs. 7-8.

Comparing all the calculated results with the test results, it can be shown that the analytical response curves calculated by the formulae are identical to the test results in wave shape and general character. The peak value of both are similar as well. Furthermore, the error range of the methods outlined in this paper are lower than that of the numerical method in general. Therefore, the analytical methods presented in this paper provide reliable and accurate results.

\section{STUDIES OF THE CHARACTERISTICS OF THE SSVR}

The formula (36) for the rectangular Duhamel method is suitable for developing some general characteristics of the SSVR.

The formula (36) shows that the response acceleration curve of the top mass is composed of a rectangular square wave 
TABLE 2

\begin{tabular}{|c|c|c|c|c|c|c|c|c|c|c|c|c|c|}
\hline \multirow{3}{*}{$\frac{\text { No. }}{1}$} & \multirow{3}{*}{$\frac{\begin{array}{c}\mathrm{T} . \\
(\mathrm{sec})\end{array}}{0.13}$} & \multirow{3}{*}{$\frac{\frac{m_{1}}{m_{2}}}{1}$} & \multirow[t]{2}{*}{$\xi$} & \multirow[t]{2}{*}{$\mu$} & \multirow{2}{*}{$\begin{array}{c}\text { Ao } \\
(g a l)\end{array}$} & \multirow{2}{*}{$\begin{array}{c}\mathrm{T} \\
(\mathrm{sec})\end{array}$} & \multicolumn{3}{|c|}{$\begin{array}{c}\text { peak of } \ddot{u}_{1}(t) \\
(\text { gal })\end{array}$} & \multirow{2}{*}{\multicolumn{2}{|c|}{ error to }} & \multirow{2}{*}{\multicolumn{2}{|c|}{ test }} \\
\hline & & & & & & & \multirow{2}{*}{$\frac{\text { test }}{497}$} & \multirow{2}{*}{$\frac{\text { TFM }}{561}$} & \multirow{2}{*}{$\frac{\text { num }}{566}$} & & & & \\
\hline & & & 0.0144 & 0.20 & 702 & 0.125 & & & & +64 & $+12.9 \%$ & +69 & $+13.9 \%$ \\
\hline 2 & 0.184 & 2 & 0.016 & 0.20 & 125.5 & 0.2 & 381 & 388 & 388 & +7 & $+1.8 \%$ & +7 & $+1.8 \%$ \\
\hline 3 & 0.065 & 2 & 0.012 & 0.20 & 331 & 0.1 & 496 & 528 & 480 & +32 & $+6.5 \%$ & -18 & $-3.2 \%$ \\
\hline 4 & 0.065 & 2 & 0.012 & 0.20 & 436 & 0.1 & 597 & 551 & 597 & -46 & $-7.7 \%$ & 0 & \\
\hline 5 & 0.184 & 2 & 0.016 & 0.20 & 209 & 0.2 & 380 & 393 & 448 & +13 & $+3.4 \%$ & +67 & $+17.8 \%$ \\
\hline 6 & 0.20 & 3 & 0.016 & 0.20 & 158 & 0.2 & 381 & 392 & 382 & +11 & $+2.9 \%$ & +1 & $+0.3 \%$ \\
\hline 7 & 0.20 & 3 & 0.016 & 0.20 & 167.5 & 0.2 & 397 & 392 & 393 & -5 & $-1.3 \%$ & -4 & $-1 \%$ \\
\hline 8 & 0.09 & 3 & 0.016 & 0.20 & 192.5 & 0.143 & 430 & 472 & 329 & +42 & $+9.8 \%$ & -101 & $-23.5 \%$ \\
\hline
\end{tabular}

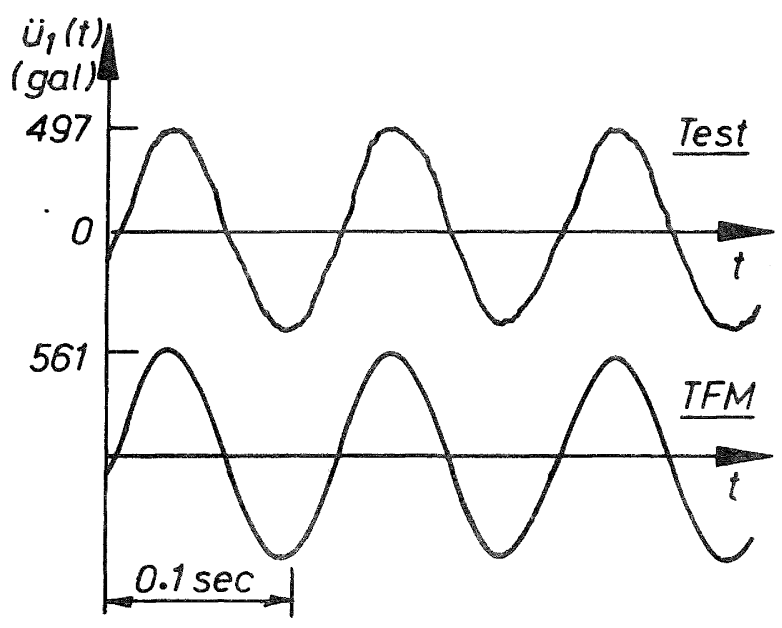

FIG. 7 COMPARISON OF SSVR (2)

$m_{1}=3.65, m_{2}=3.65, T .=0.13, \xi=0.0144$, $\mu=0.20$

input: $\mathrm{A} .=402, \mathrm{~T}=0.125$

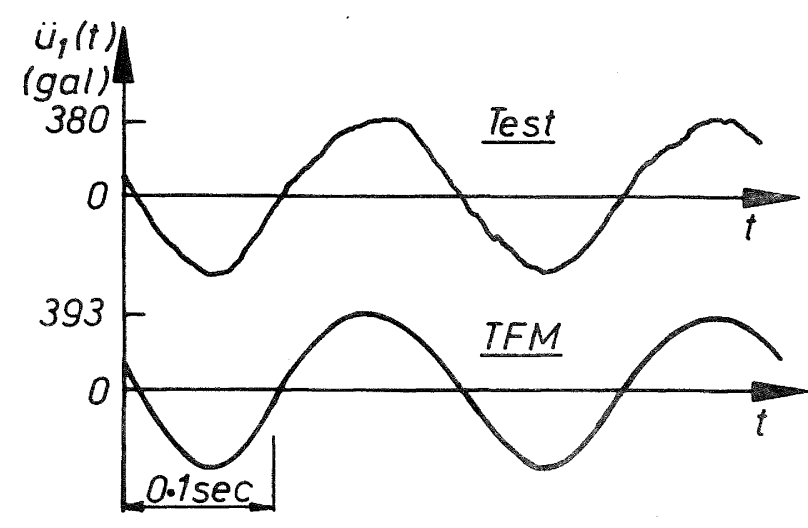

FIG. 8 COMPARISON OF SSVR (3) $m_{1}=3.65, m_{2}=3.65, T .=0, \xi=0.016$, $\mu=0.20$

input: $A .=209, T=0.20$

and in the limit the formula (36) has the form:

$$
\ddot{\mathrm{u}}_{1}(t)=(-1)^{\mathrm{N}-1} g \mu
$$

This is the extreme case where there is no base mass and agrees exactly with the formula (6).

The formula (36) also shows that the response acceleration curve of the top mass is composed of two parts: the friction curve and a concomitant free vibration with

circular frequency $n$. Every reversal of

the friction force excites a new concomitant free vibration. If $\mathrm{m}_{1}>\mathrm{m}_{2}$, then the value of $a$ is large, so these concomitant free vibrations will approach zero ouickly. In this case, the response curve tends to 


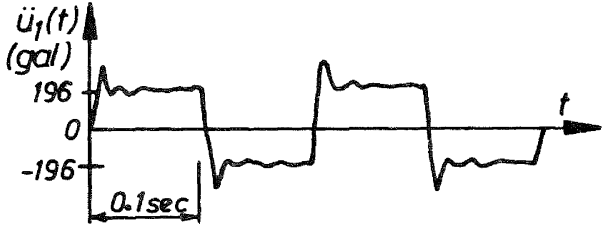

FIG. 9 RESPONSE WHEN $\mathrm{m}_{1} \gg \mathrm{m}_{2}$

be the simple forced vibration, that is:

$$
\ddot{u}_{1}(t) \doteq(-1)^{N \tilde{N}-1} g \mu
$$

Fig. 9 shows the calculated acceleration curve of SSVR for $\frac{m_{1}+m_{2}}{m^{2}}=100$

in the case of $\mu=0.2, \xi=0.05, p=5 * 2$, $\mathrm{T}=0.2$, which resembles a rectangular square wave of friction.

The operation adopted in this paper is cheap and quick, it is possible to find some general laws by means of a series of calculations. Fig. $10(a-d)$ show the relations between the peak values of SSVR and mass ratio $\mathrm{M}$ with 10 different period encounters. Fig. Il (a-b) show the sliding vibrating response spectra according to the Chinese aseismic design code (TJ-II-78). From all of these curves, some general characteristics can be found as follows:

1. The base mass obviously affects the peak value of the SSVR. When the base mass is relatively large, the response appears to be a quasi-resonance. In this case, the base sliding isolation tends to lose its beneficial aspects and even turns into the opposite.

2. In general, the peak value of SSVR diminishes with increasing top mass. If the top mass is comparatively large, the peak value of SSVR is hardly related to the dynamic characteristics of structure and the period of input, and the response is analogous to that of a rigid body.

3. The quasi-resonance of SSVR takes place not at the resonance frequency but at the moment when tie natural period of structure is higher than that of input. As the base mass decreases, this amplitude of period difference increases, while the peak value of quasi-resonance decreases.

\section{CONCLUSIONS}

The analytical methods for the SSVR calculation given in this paper can be used directly in the case of usual harmonic excitation or "disadvantageous period reciprocating input". At the same time, they can

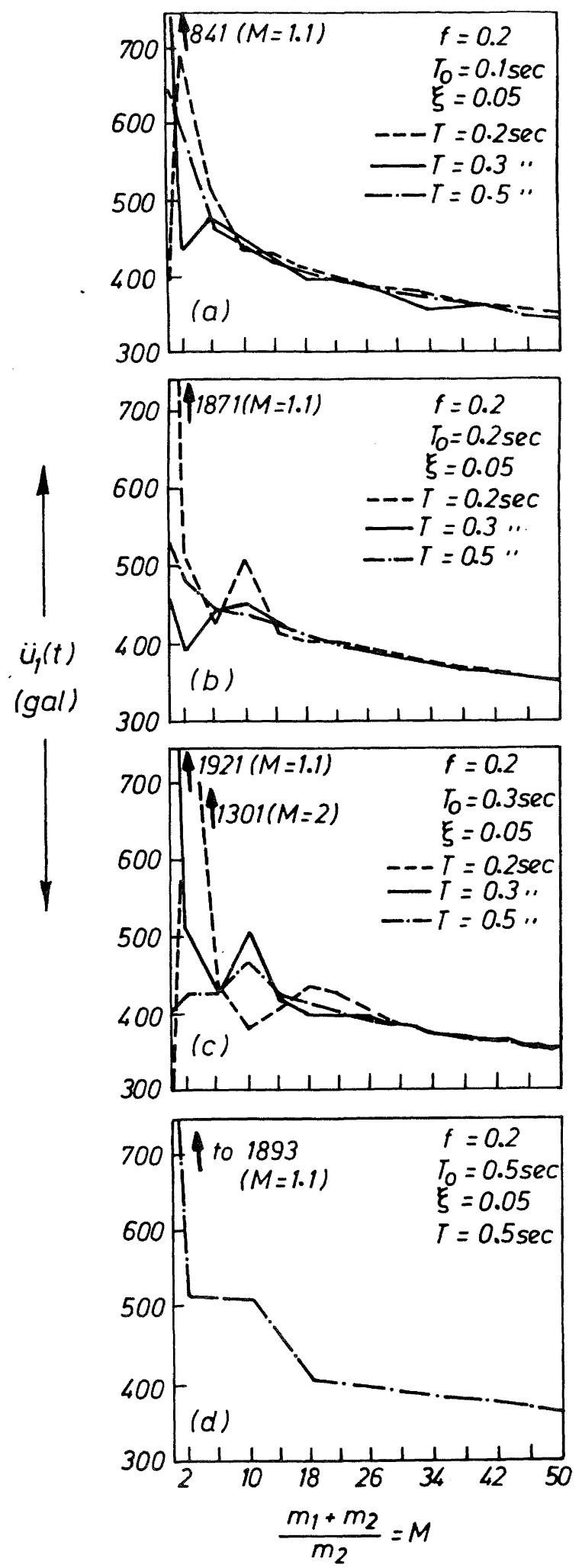

FIG. 10 RELATIONS BETWEEN RESPONSE PEAK AND MASS RATIO M 

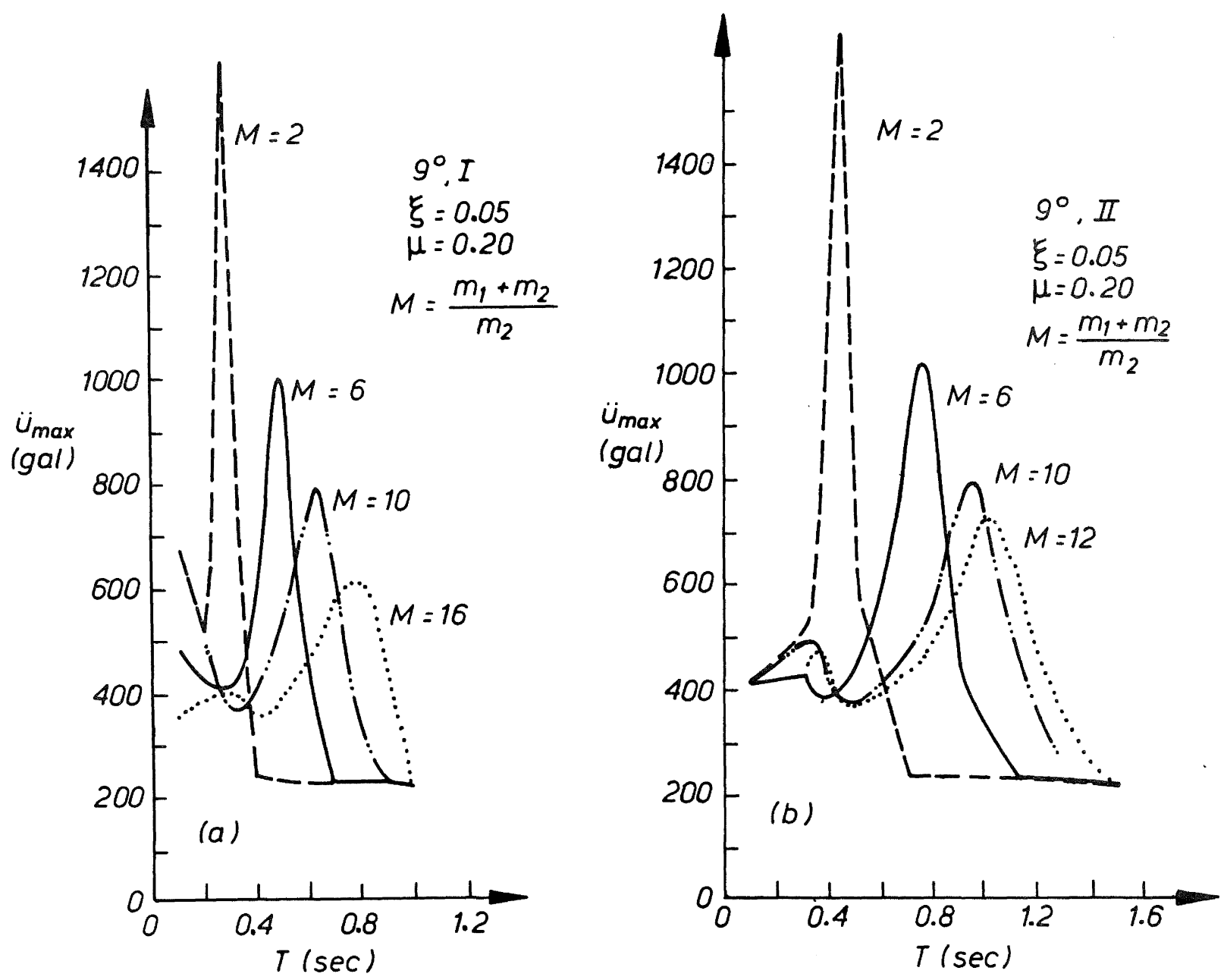

FIG. 11 SLIDING-VIBRATING RESPONSE SPECTRA

also be used to calculate SSVR under the earthquake wave with further treatment. of course, there are many researches to be done for this aim.

Through the analytical research of SSVR based on an elastic body assumption some general laws of SSVR were developed which had previously been masked by assuming rigid body motion. It also shows that the problems of base-sliding isolation are more complex than expected. In order to make full use of base-sliding isolation, it is necessary to calculate and compare SSVR practically.

\section{REFERENCES}

1. Fu Yu-an, "Anti-Overturning Analysis of High-rise Structures with Sliding Base", Master Degree Thesis, CRIBC, MMI, China, 1981.
2. Fu Yu-an, "Analysis of SlidingVibrating Responses of Elastic Structures", "Development of Earthquake Engineering', Vol.3-4, Beijing, China, 1983.

3. Fu Yu-an, "Analysis Calculating Method of Sliding-vibrating Responses to Elastic Structures under Harmonic Excitation". Research Report No. 12 of CRIBC, MMI, China, 1982 .

4. Fu Yu-an, "A Practicable Estimation for Sliding Base Isolation", Research Report No. 11 of CRIBC, MMI, China, $19 \overline{81 .}$

5. Liu Dexin, "Analysis of SlidingFriction Isolation of Structures, Master Degree Thesis, CRIBC, MMI, China, 1981. 